\title{
Commentary: Dance with me to the end of love: Serotonin and the carcinoid heart
}

\author{
Alejandro Raul Ayala, MD, ${ }^{a}$ and Tomas A. Salerno, MD $^{\mathrm{b}}$ \\ From the ${ }^{\mathrm{a} D e p a r t m e n t}$ of Medicine and ${ }^{\mathrm{b}}$ Division of Cardiothoracic Surgery, University of Miami Miller School \\ of Medicine and Jackson Memorial Hospital, Miami, Fla. \\ Disclosures: Authors have nothing to disclose with regard to commercial support. \\ Received for publication Sept 18, 2018; accepted for publication Sept 18, 2018; available ahead of print Nov 7, \\ 2018. \\ Address for reprints: Tomas A. Salerno MD, 1611 NW 12th Ave, Miami, FL 33136 (E-mail: tsalerno@med. \\ miami.edu). \\ J Thorac Cardiovasc Surg 2019;158:108-9 \\ $0022-5223 / \$ 36.00$ \\ Copyright (c) 2018 by The American Association for Thoracic Surgery \\ https://doi.org/10.1016/j.jtcvs.2018.09.065
}

Carcinoids are rare neuroendocrine tumors most frequently found in the gastrointestinal tract and bronchus. Approximately $15 \%$ of them release vasoactive hormones and neurotransmitters (serotonin, histamine, 5-hydroxytryptophan, bradykinin, tachykinins, and prostaglandins). These are inactivated by the liver, except in the presence of hepatic metastases, when they bypass the portal system. Excess production of serotonin may trigger the carcinoid syndrome (flushing, secretory diarrhea, bronchospasm, hypotension, and carcinoid heart disease). Carcinoid crisis is the term used to describe the sudden onset of the carcinoid syndrome compounded by hemodynamic instability, including tachycardia or bradycardia and hypotension.

Fibrotic carcinoid heart disease most often occurs in patients with hepatic metastases, predominantly affecting the right valvular endocardium, leaflets, papillary muscles and cords, and, less frequently, the pulmonary arteries and aorta. Pulmonary inactivation of vasoactive amines spares the left cardiac valvular apparatus, except in the presence of a right-to-left shunt. Hepatic metastases as well as higher urinary and plasma serotonin values predict carcinoid cardiac disease, but not all patients with liver involvement have tumors that are evident before operation. Furthermore, direct venous drainage of the ovary into the inferior vena cava explains why some women with primary ovarian carcinoid may have carcinoid heart disease develop in the absence of hepatic metastases. Rarely, metastatic disease of the heart is detected by echocardiography.

Long-term treatment for carcinoid heart disease includes pharmacologic therapy, hepatic artery embolization, chemoembolization for hepatic metastases, and surgery. ${ }^{1}$ Treatment with somatostatin analogs may mitigate or ameliorate symptoms related to the carcinoid syndrome, but it does not prevent the development of cardiac

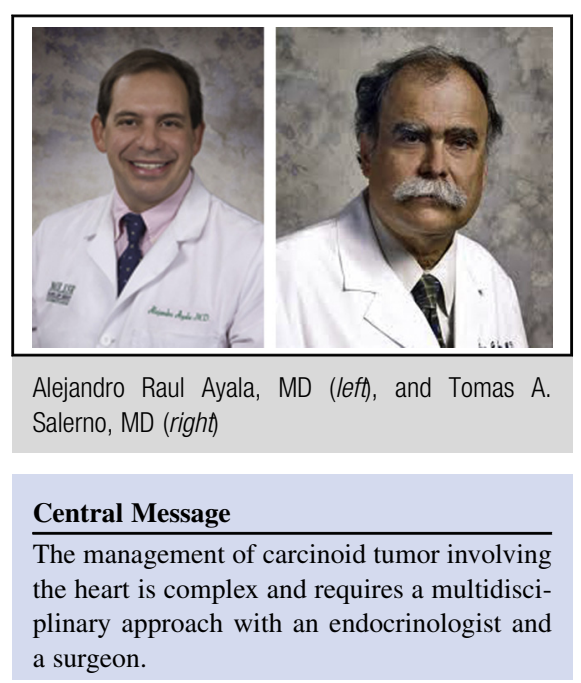

See Article page 99.

disease. $^{2}$ A number of patients may have symptomatic tricuspid and pulmonic valve disease develop, requiring repair or replacement and closure of patent foramen ovale. The technical aspects of these procedures are well known. Medications (vasopressors), surgery, acute stress, contrast agents, and anesthetic induction can all precipitate vasoactive amine release. Preoperative assessment and therapy focus on prevention of carcinoid crisis and perioperative complications.

The timely article by Nguyen and colleagues ${ }^{3}$ in this issue of the Journal, covering more than 3 decades and 240 patients, provides useful and detailed insights on this matter. Reflecting established practice, most patients $(92 \%)$ received either long- or short-acting octreotide before the operation. The protocol for those with advanced disease included preoperative hospitalization, followed by intraoperative and postoperative $100-$ to $200-\mu \mathrm{g} / \mathrm{h}$ infusions of short-acting octreotide. An additional dose of $1000 \mu \mathrm{g}$ was administered if the patient showed signs of carcinoid crisis. A few important conclusions can be derived from the study. First, it is clear that a multidisciplinary approach is necessary to deal with such patients in a safe and effective manner. Second, early mortality from 1985 to 1994 was $29 \%$, dropping to 7\% from 1995 to 2004 and 5\% currently. Nguyen and colleagues ${ }^{3}$ point out that the causes of such improvements are difficult to dissect and may include advances in surgical techniques, new diagnostic tools (eg, gallium 68 positron emission tomographic computed tomography), 
patient selection, adjustments of perioperative somatostatin analog protocols, and overall improved perioperative care. Modifications related to somatostatin analog administration that have occurred with time may have well played an important role. This is highlighted by other similar studies suggesting that the combination of long-acting octreotide with short-acting boluses may not be as effective as a prophylactic continuous infusion preceded by a bolus. ${ }^{4,5}$ Finally, reaffirming existing literature, survival seems to be predicted by functional status and comorbidities. ${ }^{4}$

Carcinoid heart disease remains a formidable challenge, despite medical and surgical improvements. Given its rarity, it is unlikely that we will have access to randomized studies comparing perioperative protocols any time soon. Patient selection, careful preoperative planning, and protocols for perioperative continuous somatostatin infusion regimens, all under the care of a multidisciplinary team, are paramount for success.

\section{References}

1. Connolly HM, Nishimura RA, Smith HC, Pellikka PA, Mullany CJ, Kvols LK Outcome of cardiac surgery for carcinoid heart disease. J Am Coll Cardiol. 1995:25:410-6.

2. Møller JE, Connolly HM, Rubin J, Seward JB, Modesto K, Pellikka PA, et al. Factors associated with progression of carcinoid heart disease. N Engl J Med. 2003 348:1005-15.

3. Nguyen A, Schaff HV, Abel MD, Allen Luis S, Lahr BD, Halfdanarson TR, et al Improving outcome of valve replacement for carcinoid heart disease. $J$ Thorac Cardiovasc Surg. 2019;158:99-107.e2.

4. Massimino K, Harrskog O, Pommier S, Pommier R. Octreotide LAR and bolus octreotide are insufficient for preventing intraoperative complications in carcinoid patients. J Surg Oncol. 2013;107:842-6.

5. Woltering EA, Wright AE, Stevens MA, Wang YZ, Boudreaux JP, Mamikunian G, et al. Development of effective prophylaxis against intraoperative carcinoid crisis. J Clin Anesth. 2016;32:189-93. 